\title{
Erratum to: Combination of anxiety and depression is associated with an increased headache frequency in migraineurs: a population-based study
}

Kyungmi Oh', Soo-Jin Cho ${ }^{2}$, Yun Kyung Chung ${ }^{3}$, Jae-Moon Kim ${ }^{4}$ and Min Kyung Chu ${ }^{5^{*}}$

\section{Erratum}

After publication of the original article [1], the authors noticed that the article contained some errors in the data presented within Table 1, Figs. 1 and 2, and Tables 2 and 3.

The corrected versions are given in this erratum. The main findings of the original article are not affected after this correction. The authors apologise for any inconvenience this has caused.

\footnotetext{
Author details

'Department of Neurology, Korea University Guro Hospital, Korea University School of Medicine, Seoul, Korea. ${ }^{2}$ Department of Neurology, Dongtan Sacred Heart Hospital, Hallym University College of Medicine, Hwaseong, Korea. ${ }^{3}$ Department of Occupational and Environmental Medicine, Sacred Heart Hospital, Hallym University College of Medicine, Anyang, Korea. ${ }^{4}$ Department of Neurology, Chungnam National University, College of Medicine, Daejeon, Korea. ${ }^{5}$ Department of Neurology, Sacred Heart Hospital, Hallym University College of Medicine, Anyang, Korea.
}

Published online: 19 April 2016

\section{Reference}

1. Oh K, Cho S-J, Chung YK, Kim JM, Chu MK. Combination of anxiety and depression is associated with an increased headache frequency in migraineurs: a population-based study. BMC Neurol. 2014;14:238. doi:10.1186/s12883-014-0238-4

\footnotetext{
* Correspondence: chumk@hallym.ac.kr

${ }^{5}$ Department of Neurology, Sacred Heart Hospital, Hallym University College of Medicine, Anyang, Korea

Full list of author information is available at the end of the article
}

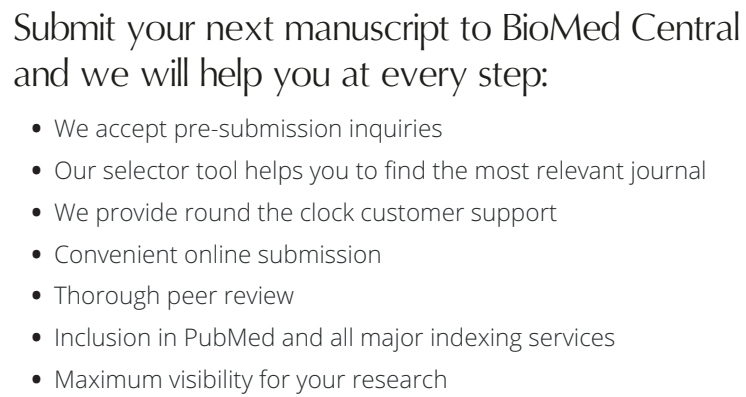

- We accept pre-submission inquiries

- Our selector tool helps you to find the most relevant journal

- We provide round the clock customer support

- Convenient online submission

- Thorough peer review

- Inclusion in PubMed and all major indexing services

- Maximum visibility for your research

Submit your manuscript at www.biomedcentral.com/submit 
Table 1 Sociodemographic distribution of all survey participants, the total Korean population, and of cases identified as migraine, anxiety and depression

\begin{tabular}{|c|c|c|c|c|c|c|}
\hline & $\begin{array}{l}\text { Survey participants } \\
N(\%)\end{array}$ & $\begin{array}{l}\text { Total population } \\
N(\%)\end{array}$ & $P$ & $\begin{array}{l}\text { Migraine N, } \\
\%(95 \% \mathrm{Cl})\end{array}$ & $\begin{array}{l}\text { Anxiety N, } \\
\%(95 \% \mathrm{Cl})\end{array}$ & $\begin{array}{l}\text { Depression N, } \\
\%(95 \% \mathrm{Cl})\end{array}$ \\
\hline Men & $1345(49.3)$ & $17,584,365(50.6)$ & $0.854^{\mathrm{a}}$ & $36,2.7(1.8-3.5)$ & 109, 8.1 (6.6-9.6) & $43,3.2(2.3-4.2)$ \\
\hline Women & $1350(50.7)$ & $17,198,350(49.4)$ & & $107,7.9(6.5-9.4)$ & $159,11.8(10.1-13.5)$ & $73,5.4(4.2-6.6)$ \\
\hline \multicolumn{7}{|l|}{ Age } \\
\hline $19-29$ & $542(20.5)$ & $7,717,947$ (22.2) & $0.917^{\mathrm{a}}$ & $25,4.5(2.7-6.2)$ & $53,9.6(7.2-12.1)$ & $23,4.1(2.5-5.8)$ \\
\hline $30-39$ & 604 (21.9) & $8,349,487(24.0)$ & & $42,7.0(4.9-9.1)$ & $51,8.7(6.4-11.0)$ & $32,5.4(4.6-7.3)$ \\
\hline $40-49$ & $611(23.1)$ & $8,613,110(24.8)$ & & $39,6.5(4.5-8.4)$ & $67,11.0(8.5-13.5)$ & $24,4.0(2.5-5.5)$ \\
\hline $50-59$ & $529(18.9)$ & $6,167,505$ (17.7) & & $22,4.1(2.4-5.9)$ & $53,9.9(7.3-12.5)$ & $22,4.2(2.5-6.0)$ \\
\hline $60-69$ & 409 (15.6) & $3,934,666(11.3)$ & & $15,3.9(2.0-5.7)$ & $14,10.8(7.8-13.8)$ & $15,3.7(2.0-5.5)$ \\
\hline \multicolumn{7}{|l|}{ Size of residential area } \\
\hline Large city & $1248(46.3)$ & $16,776,771(48.2)$ & $0.921^{a}$ & $76,6.1(4.8-7.5)$ & $130,10.4(8.7-12.1)$ & $57,4.6(3.4-5.7)$ \\
\hline Medium-to-small city & $1186(44.0)$ & $15,164,345(43.6)$ & & $48,4.0(2.9-5.2)$ & $112,9.5(7.8-11.2)$ & $47,4.0(2.9-5.1)$ \\
\hline Rural area & $261(9.7)$ & 2,841,599 (8.2) & & $19,7.4(4.2-10.6)$ & $26,10.0(6.3-13.6)$ & $12,4.7(2.1-7.3)$ \\
\hline \multicolumn{7}{|l|}{ Education level } \\
\hline Middle school or less & $393(14.9)$ & $6,608,716(19.0)$ & $0.752^{a}$ & $22,5.5(4.2-7.7)$ & $55,13.9(10.5-17.4)$ & $20,5.2(3.0-7.4)$ \\
\hline High school & $1208(44.5)$ & $15,234,829(43.8)$ & & $60,5.0(3.8-6.3)$ & $111,9.2(7.5-10.8)$ & $49,4.1(3.0-5.2)$ \\
\hline College or more & 1068 (39.6) & $12,939,170(37.2)$ & & $60,5.6(4.3-7.0)$ & $100,9.5(7.7-11.2)$ & $47,4.4(3.2-5.7)$ \\
\hline Not responded & $26(9.6)$ & & & 1, $3.8(0.0-11.8)$ & $2,8.0(0.0-18.0)$ & $0,0.0(0.0-0.0)$ \\
\hline Total & $2695(100.0)$ & $34,782,715(100.0)$ & & $143,5.3(4.5-6.2)$ & $268,10.0(8.8-11.1)$ & $116,4.3(3.6-5.1)$ \\
\hline
\end{tabular}

${ }^{a}$ Compared gender, age group, size of residential area, and educational level distributions between the sample of the present study and total population of Korea

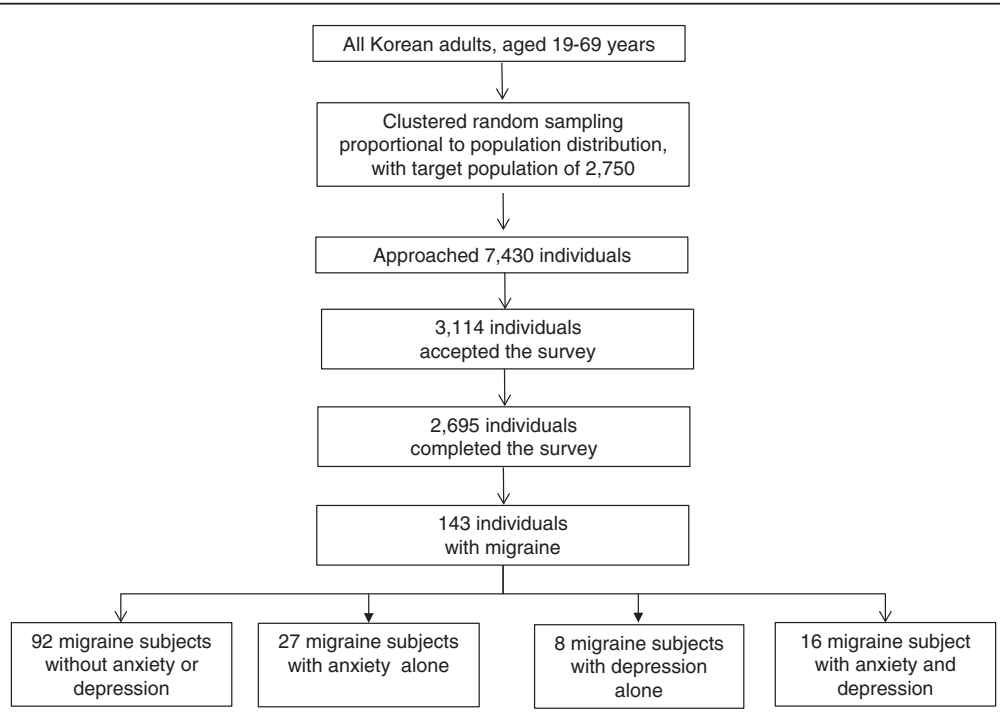

Fig. 1 Flow chart depicting the participation of subjects in the Korean Headache-Sleep Study 


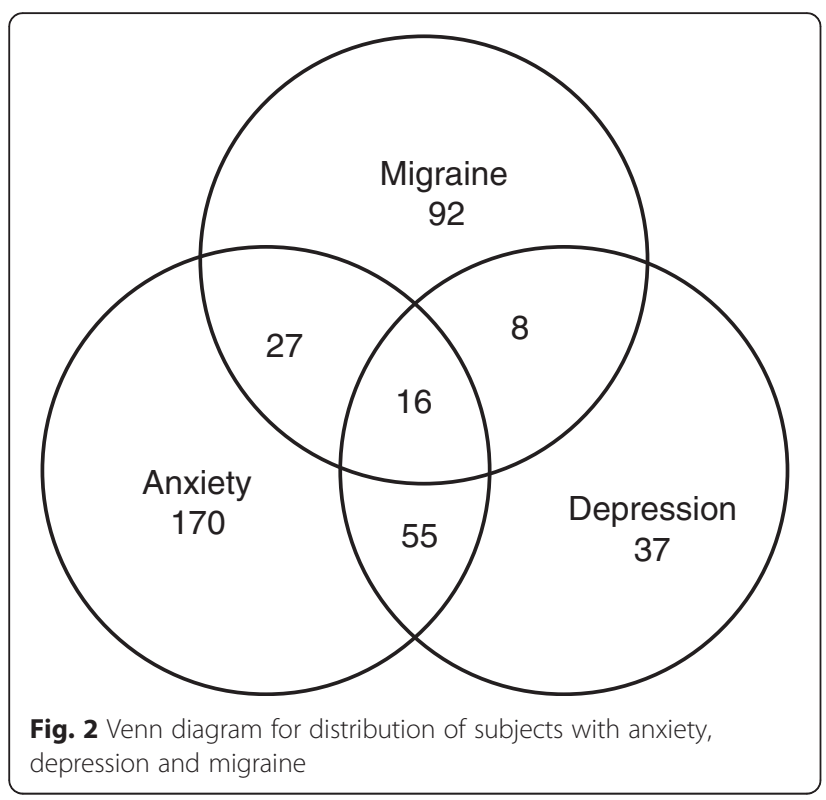

Table 2 Demographics, headache characteristics and associated symptoms of migraineurs according to anxiety and depression

\begin{tabular}{|c|c|c|c|c|c|}
\hline & $\begin{array}{l}\text { Migraine subjects without } \\
\text { anxiety or depression, } N=92\end{array}$ & $\begin{array}{l}\text { Migraine subjects with } \\
\text { anxiety alone, } N=27\end{array}$ & $\begin{array}{l}\text { Migraine subjects with } \\
\text { depression alone, } N=8\end{array}$ & $\begin{array}{l}\text { Migraine with anxiety } \\
\text { and depression, } N=16\end{array}$ & $P$-value \\
\hline \multicolumn{6}{|l|}{ Demographics } \\
\hline Mean age \pm SD (years) & $40.4 \pm 11.4$ & $47.2 \pm 14.5$ & $42.1 \pm 15.7$ & $44.5 \pm 18.4$ & 0.131 \\
\hline Women, $N(\%)$ & $71(75.5)$ & $21(77.8)$ & $7(77.8)$ & $13(76.5)$ & 0.995 \\
\hline \multicolumn{6}{|l|}{ Headache characteristics } \\
\hline Unilateral pain, $N(\%)$ & $57(60.6)$ & 15 (53.6) & $4(44.4)$ & $7(41.2)$ & 0.409 \\
\hline Pulsating quality, N (\%) & $72(76.6)$ & $21(77.8)$ & $7(77.8)$ & $12(70.6)$ & 0.950 \\
\hline Moderate-to-severe severity, N (\%) & $72(75.8)$ & $22(81.5)$ & $8(88.9)$ & $17(100.0)$ & 0.119 \\
\hline Aggravation by movement, $N(\%)$ & $60(63.8)$ & $21(77.8)$ & $5(55.6)$ & $15(88.2)$ & 0.121 \\
\hline \multicolumn{6}{|l|}{ Associated symptoms } \\
\hline Nausea, $N(\%)$ & $84(88.4)$ & $23(82.1)$ & $9(100.0)$ & $15(88.2)$ & 0.544 \\
\hline Vomiting, $N(\%)$ & $33(34.7)$ & $15(53.6)$ & $3(33.3)$ & $8(47.1)$ & 0.288 \\
\hline Photophobia, N (\%) & $55(57.9)$ & $19(70.4)$ & $1(11.1)$ & $11(64.7)$ & 0.017 \\
\hline Phonophobia, N (\%) & $63(67.0)$ & $21(77.8)$ & $6(66.7)$ & $13(76.5)$ & 0.668 \\
\hline Osmophobia, N (\%) & $42(44.7)$ & $13(48.1)$ & $4(44.4)$ & $9(46.3)$ & 0.930 \\
\hline
\end{tabular}


Table 3 Frequency, severity and impact of headache according to migraineurs' anxiety and depression status

\begin{tabular}{|c|c|c|c|c|c|c|}
\hline & $\begin{array}{l}\text { Migraine subjects without } \\
\text { anxiety or depression, } \\
N=92\end{array}$ & $\begin{array}{l}\text { Migraine subjects } \\
\text { with anxiety alone, } \\
N=27\end{array}$ & $\begin{array}{l}\text { Migraine subjects } \\
\text { with depression alone, } \\
N=8\end{array}$ & $\begin{array}{l}\text { Migraine subjects } \\
\text { with anxiety and } \\
\text { depression, } N=16\end{array}$ & $P$-value* & $\begin{array}{l}\text { Post hoc analysis } \\
\text { with Bonferroni's } \\
\text { correction }\end{array}$ \\
\hline & Median (25 \%-75 \%) & Median (25 \%-75\%) & Median (25 \%-75\%) & Median (25 \%-75\%) & & \\
\hline \multirow{6}{*}{$\begin{array}{l}\text { Frequency per } \\
\text { month }\end{array}$} & \multirow[t]{6}{*}{$1.0(0.3-3.0)$} & \multirow[t]{6}{*}{$2.0(1.0-5.0)$} & \multirow[t]{6}{*}{$1.0(0.3-4.0)$} & \multirow[t]{6}{*}{$8.0(2.5-21.0)$} & \multirow[t]{6}{*}{$<0.001$} & 1 vs. $2=0.596$ \\
\hline & & & & & & 1 vs. $3=1.000$ \\
\hline & & & & & & 1 vs. $4<0.001$ \\
\hline & & & & & & 2 vs. $3=1.000$ \\
\hline & & & & & & 2 vs. $4=0.003$ \\
\hline & & & & & & 3 vs. $4=0.001$ \\
\hline \multirow{6}{*}{$\begin{array}{l}\text { VAS score for } \\
\text { pain intensity }\end{array}$} & \multirow[t]{6}{*}{$6.0(5.0-7.0)$} & \multirow[t]{6}{*}{$7.0(6.0-8.0)$} & \multirow[t]{6}{*}{$7.0(6.0-8.0)$} & \multirow[t]{6}{*}{$7.0(5.0-9.0)$} & \multirow[t]{6}{*}{$<0.001$} & 1 vs. $2=0.011$ \\
\hline & & & & & & 1 vs. $3=0.824$ \\
\hline & & & & & & 1 vs. $4=0.018$ \\
\hline & & & & & & 2 vs. $3=1.000$ \\
\hline & & & & & & 2 vs. $4=1.000$ \\
\hline & & & & & & 3 vs. $4=1.000$ \\
\hline \multirow[t]{6}{*}{ HIT-6 score } & \multirow[t]{6}{*}{$50.0(46.0-58.0)$} & \multirow[t]{6}{*}{$57.0(49.0-60.8)$} & \multirow[t]{6}{*}{$62.0(52.0-70.5)$} & \multirow[t]{6}{*}{$64.0(61.0-67.0)$} & \multirow[t]{6}{*}{$<0.001$} & 1 vs. $2=0.074$ \\
\hline & & & & & & 1 vs $.3=0.004$ \\
\hline & & & & & & 1 vs. $4<0.001$ \\
\hline & & & & & & 2 vs. $3=0.545$ \\
\hline & & & & & & 2 vs. $4=0.005$ \\
\hline & & & & & & 3 vs. $4=1.000$ \\
\hline
\end{tabular}

HIT-6: Headache Impact Test-6; VAS: Visual Analogue Scale

*Kruskal-Wallis one-way analysis of variance test among the four groups: migraine subjects without anxiety or depression, migraine subjects with anxiety alone, migraine subjects with depression alone and migraine subjects with anxiety and depression

1: migraine subjects without anxiety or depression; 2: migraine subjects with anxiety alone; 3 : migraine subjects with depression alone; 4 : migraine with anxiety and depression 